molekulare Säuren, aber keine Stearinsäure, und ein Phytosterin. Die quantitative Zusammensetzung des Daturaöls ist annähernd: Palmitinsäure $10 \%$, Daturinsäure $2,5^{0} \%$, Ölsäure $62 \%$, Linolsäure $15 \%$, Unverseifbares (Phytosterin) $1 \%$, Glycerin $9,6 \%$. Die Linolsäure gab bei der Bromierung ausschljeßlich die bei $117^{\circ}$ schmelzende Tetrabromstearinsäure; bei der Oxydation aber zwei Modifikationen der Sativinsäure, eine bei $162-163^{\circ}$ und eine bei $173^{\circ}$ schmelzende. Führt man die Tetrabromstearinsäure wieder in Linolsäure über und oxydiert, so erbält man nur die niedriger schmelzende $(\alpha-)$ Sativinsäure, deren Unterschied von der höher schmelzenden ( $\beta$-) Sativinsäure wahrscheinlich auf Stereoisomerie beruht.

G. Sonntag.

\title{
Versammlungen, Tagesneuigkeiten etc.
}

Bericht iiber die Tagungen der Vereinigung Württembergischer Nahrungsmittelchemiker in Stuttgart am 11. und 25. März 1916.

Die Vereinigung Württembergischer Nahrungsmittelehemiker hielt am 11. und 25. März $z$ wei auferordentliche Tagungen ab zur Besprechung von kriegswirtschaftlichen Fragen, die mit der Nahrungsmittelchemie in Verbindung stehen. Die Mitglieder waren fast vollzählig erschienen, ein Beweis, daß 3 die Tagungen einem Bedürfnis entsprachen.

Bei der 1. Tagung vom 11. März 1916 standen folgende Gegenstände zur Beratung:

1. Konserven für Feldpostsendungen (Liebesgaben). Referent Dr. GaabLudwigsburg: Es wurde in erster Linie der hohe Preis vieler Fleischkonserven für Feldpostpackung bemängelt. Die Versammlung war der Ansicht, dak der Nahrungsmittelchemiker durch seine Untersuchungen eine Qualitätsbeurtellang vorzunehmen und lediglich die Grundlagen zu einer genauen Preisprüfung zu liefern imstande sei, sodaß dann auf Grund einer kaufmännischen Wertberechnung übermäßjigen Preistreibereien im Sinne der Bundesratsverordnung wirksam entgegengetreten werden könne.

2. Neue Konservierungsmittel. Referent Dr. Schmiedel-Stuttgart: Infolge der Beschlagnahme des Salpeters und des für die Herstellung von Benzoesäure verwendeten Toluols sind während des Krieges salpetrigsaure Salze zur Fleischpökelung und Metakresotin. säure als Konservierungsmittel in Aufnahme gekommen. Der Redner betonte die günstige Beurteilung der Metakresotinsäure durch die Gutachter Schotitelius und Lehmann und machte darauf aufmerksam, daß sie mit Eisenchlorid wie die Salicylsäure eine Violettfärbung gebe, sich aber von dieser durch den Schmelzpunkt unterschenden lasse. Die Versammlung war der Ansicht, daß eine Beanstandung bezw. Verurteilung wegen Zusatzes von salpetrigsauren Salzen zur Fleischpökelung im Hinblick auf die limpfehlung von Prof. Glage schwerlich möglich sein würde. (Bemerkt sei, daß nach einem inzwischen erschienenen Erraßs des Preuß3. Ministeriums sich die wissenschaftliche Deputation $f$. d. Medizinalwesen dahin geäufzert hat, dá̧ gegen die Verwendung klein er Nitritmengen, wie sie z. B. bei der Herstellung von Pökelfleisch in Betracht kommen, keine Bedenken bestehen, daß aber die Verwendung von Nitriten zur Wurstbereitung für un zulässig zu erachten sei.)

3. Veränderungen in der Zusammensetzung der Wurstwaren während der Kriegszeit. Referent Dr. Seel-Stuttgart betonte besonders die Verschiebung des Verhältnisses von magerem Fleisch zu Fett in den Warstwaren zugunsten des ersteren. Die Würste sind jetzt gegenüber den Verbaltnissen in Friedenszeiten sehr fettarm und sehr wasserreich; es ist deshalb die Festsetzung einer Höchstgrenze für den Wassergehalt der Kriegs- und Friedenswürste sebr erwünscht. Der Nährwert der Kriegswarste ist bedeutend niedriger als der der Friedenswürste. Die Versammlung beschliekt bei der Bestimmung des Nährwertes der Würste und anderer Nahrungsmittel möglichst die von der Militärverwaltung benutzten Grundsätze anzuwenden (ausnutzbare Nährwerteinheiten in Kalorien). - Vergl, diese Zeitschrift 1916, 32, 29.

4. Erfahrangen bei Untersachang von Seifen während der Kriegszeit. Referent Oberapotheker $\nabla$ o h rin g e r berichtete aber die große Preissteigerung und über die vielen Schwindeleien im Seifenhandel. Die in den Tageszeitungen massenhaft angepriesenen Seifen enthalten oft nur $6-7 \%$ Fettsäure, dafür aber bis zu $90 \%$ Wasser und andere wertlose Stoffe; ihr Preis ist im Verhältnis zu ihrem wirklichen Wert um das Mehrfache zu hoch. Die Mehrzabl der versammelten Mitglieder bestätigte die Beobachtungen des Redners. Prof. Rau hob hervor, die jetzt angepriesenen billigen Schmierseifen seien häufig nichts anderes als ein aus 
Kernseife unter Zusatz von Soda und Wasserglas bergestellter Seifenleim. Die Bezeichnung Schmierseife für solche Erzengnisse sei auch an sich falsch, da man unter Schmierseife eine Kaliseife verstehe. Die Versammlung war der Ansicht, dafs versucht werden soll, den Erlaß einer den Seifenverkauf regelnden Verordnung zu erreichen. Dr. Alpers wurde damit betraut, eine diesbezügliche Eingabe an das stellv. Generalkommando ẌIIL. Armeekorps zu entwerfen, die inzwischen abgegangen ist. - Im Anschluk an die Besprechungen hob Dr. Benz die Irreführang des Publikums durch die Veröffentlichung unbrauchbarer Rezepte für die Herstellung von Seife hervor. Ess schlof sich hieran noch eine Aussprache über Seifenersatzmittel an.

5. Probenahme und Fettbestimmung bei Käse. Referent Prof. Dr. Rau. Stuttgart: Die Bundesratsverordnung und Ministerialverfügang über den Verkehr mit Käse haben zahlreiche Käsefettbestimmungen zwecks Feststellung der Käsesorte notwendig gemacht. Dr. Seitter. Olm berichtete über grofe Unterschiede im Fettgehalt bei den einzelnen Käselaiben und schlug einheitlicheres Vorgehen in der Art der Untersuchung und besonders der Probenahme vor. Die Versammlung war der Ansicht, daf zur Beurteilung des Fettgehaltes einer Käselieferung die zu untersuchende Probe durch Mischen kleiner, aus mehreren Laiben entnommener Proben gewonnen werden soll, um eine gate Durchschnittsprobe zu erzielen. Bei der Beurteilung grosaer Käsesendungen worde es zur Vermeidung allza hoher Untersuchungskosten für zweckmäßig erachtet, in bezag auf die Anzahl der zu untersuchenden Proben ähnlich za verfahren, wie dies in der zollamtlichen Anweisung zur Fettprobenentnahme vorgesehen ist. - Im AnschlnG hieran berichtete Prof. $R$ au auf Anregang des abwesenden Dr. Teichert über Milchersatz "Sahna" und einen bulgarischen Käseersatz. Beide Präparate wurden von der Versammlung abfällig beurteilt.

6. Beobachtungen über fadenziehendes Brot. Referent Dr. Alpers.Tübingen: Die Erscheinung des Fadenziehens des Brotes wurde im letzten Sommer sehr häufig beobachtet; es sind beträchtliche Mengen an Brot durch diese Brot-Krankheit verloren gegangen. Es wurde deshalb für zweckmäkig erachtet, Bäcker und Hausfrauen an die Mittel zur Verhütung dieser Verluste zu erinnern. Dr. Schmiedel übernahm es, einen belehrenden Artikel für die Bäckerfachzeitung zu verfassen, was inzwischen geschehen ist.

7. Nachweis der verschiedenen Stärkearten durch die sog. Schwarzweif-rot-Eärbung. Referenten Dr. Sander und Dr. Mezger-Stuttgart: Dr. Sander ermittelte, daß 3 die von der Getreideverwertungsstelle in Berlin zar Unterscheidung von Kartoffel- und Getreidestärke empfohlene sog. Schwarz-weif-rot-Farbstofflosung, deren Zusammensetzung von der betr. Gesellschaft geheim gehalten wird, aus einer Auflösung von Kongorot und Tusche unter Zusatz von Karbolsäure und Ammoniak besteht. Dr. Mezger betonte, dẩ nach seiner Erfahrung die Farbung eigentlich überflüssig sei, da ausschlaggebend bleibe für die Erkennung der Getreidestärkekörner die bei stärkerem Abblenden an einzelnen Exemplaren stets sichtbare zentrale Schichtung derselben. Im übrigen färben sich die Getreidestärkekörner nach Dr. Mezger's Beobachtungen mit der Farbstofflösung nicht عchwarz, sondern höchstens gran, während die Kartoffelstärkekörner weiß bleiben und die Kleisterzellen aus der Kartoffel eine Rosafärbung annehmen. Über die genaue Zusammensetzung der Farbstofflösung soll an anderer Stelle noch näher berichtet werden. - Diese Mitteilungen führten zu einer lebhaften Aussprache über Gebäckuntersuchungen und über die kriegsgesetzlichen Bestimmungen im Verkehr mit Backwaren. Dje Ergebnisse der Anssprache baben jedoch größtenteils nur Bedeutung fïr die württembergischen Verbältnisse; es sei deshalb von einer Auffuhrung der Einzelheiten abgesehen. Auf Vorschlag von Dr. Mezger wurde eine Eingabe an die Landesgetreidestelle, betr. ein Verbot der Verwendung von Gersten- und Malzmehl für die Herstellung von Kuchen beschlossen. Er betonte, daß̉ Weizen- und Roggenmehl zur Kuchenbereitung zwar verboten, daßs aber in letzter Zeit Auslandsgerstenmehl an Bäcker zur Kuchenbereitung abgesetzt worden sel. Im Hinblick auf die Schwierigkeit der Unterscheidung von Gersten-, Weizen- und Roggenmehl in der fertigen Backware sei so die Kontrolle illnsorisch geworden.

8. Salatolersatzmittel. Referent Prof. Philip berichtete, daß die als Salatôlersatz in den Handel gebrachten Erzeugnisse in der Regel konservierte Karrageenschleime seien. Es kommen jetzt Erzeugnisse auf den Markt, die keimfrei und haltbar sind. Dr. Alpers und Dr. Werner sprachen sich entschieden gegen die Herstellung und den Verkauf derartiger Salatölorsatzmittel aus. Die Versammlung war der Ansicht, daß, wenn das Erzeugnis unter einer einwandfreien Bezeichnung, wie z. B. *olfreier Salatzusatz, dureh besondere Zusätze baltbar gemacht" zu einem angemessenen Preise in den Handel komme, es von der Nahrungsmittelkontrolle nicht beanstandet werden könne.

Auf Anregung von Dr. Seel wurde beschlossen, bei den militärischen Behörden die Herausgabe eines Exemplares der Lieferungsbedingungen für Nahrungs- und Genufmittel, die während des Krieges von Dr. Me z ger einer Durcbsicht unterzogen worden seien, im Interesse einer einheitlichen Beurteilung der Lebensmittel seitens der Zivil- und Militärbehörden zu erwirken. 
Nach etwa 4-stündiger Verhandlung vertagte sich die Versammlung bis zum 25. März 1916, da noch verschiedene Gegenstände zur Beratung standen.

\section{Tagung am 25. März 1916.}

Es erfolgte die Verlesung des Protokolls der Sitzung vom 11. März 1916, sowie der beschlossenen Eingaben.

Die Besprechungen über die backverordnungen wurden noch fortgesetzt. Auf Antrag von Dr. Mezger wurde der Beschlufi gefaß̧t, an die Landesgetreidestelle mit der Bitte heranzutreten, ein Verbot der Verwendung von Brotbrösel für die Herstellung von Kuchen zu erlassen. Der Referent betonte, daf ohne ein solches Verbot die Bäcker eben nach wio vor Getreidemehl za Kuchen zu verwenden in der Lage seien, obne daf ihnen dies auf Grund der mikroskopischen Dntersuchung der betreffenden Backwaren in einwandfreier Weise nachgewiesen werden könne. Lediglich zum Bestreuen von Obstkuchen sollen Brösel zugelassen werden. Der Referent warde mit der Ausarbeitung eines diesbezüglichen Entwurfs beauftragt. Dr. Benz gab bekannt, daßi er den Erlas einer Zusammenfassung der jetzt noch gültigen Vorschriften, betr. den Verkehr mit Mehl und Backwaren für Württemberg an makgebender Stelle beantragt habe. Die Versammlung ist einstimmig der Ansicht, da 3 die Bekanntgabe einer solchen Zusammenstellung für Gewerbetreibende und Kontrollbehörden gleich wünschenswert sei. Punkte:

Anßer einigen wenigen wichtigen Anfragen betraf der Rest der Tagesordnung folgende

1. Ersatzmittel, Referent Dr. Seel-Stuttgart. Seine interessanten Ausführungen über die verschiedenen Ersatzmittel auf nahrungsmittelchemischem, pharmazeutischem und technischem Gebiete sollen demnächst als besondere Veröffentlichung erscheinen. Aus der sich anschliefzenden Diskussion sei folgendes hervorgehoben: Dr. Beitter-Göppingen: Nach seinen Beobachtungen gelangen die Salatölersatzmittel vielfach direkt aus den Vorratsgefäßen in Flaschen ohne Etikette, also ohne jede Deklaration zum Verkauf; es werden vielfach, sei es absichtlich, sei es aus Unkenntnis seitens der Verkäufer, diese Produkte schlechtweg als "Salatöl* bezeichnet. Die Nahrungsmittelpolizei sei auferstande, einer solchen Unterschiebung entgegenzutreten. - Dr. Mezger empfiehlt geheime Ankäufe, um Schwindeleien mit fettfreiem Salatzusatz zu unterdrücken. - Dr. Benz vertritt die Ansicht, daß bei der Stellungnahme, die die Versammlung dem Salatölersatz gegenüber einnehme, das Moment der Täuschung, auf welches dic Fabrikanten $z$ weifelsohne ausgehen, zu wenig berücksichtigt sei. Er teilte noch mit, dafi ein von ihm untersuchter Salatölersatz 7,3\% Wasserglas enthalten habe.

2. Bieruntersuchungen während der letzten Kriegsmonate. Referent: Herr Jesser (nach Analysen von Dr. Greiner am Stuttgarter Städt. Laboratorium). Der Vergärungsgrad der untersuchten Biere schwankte $z$ wischen 39 und 60 , der Alkoholgehalt $z$ wischen 1,3 und $3,1 \%$, der Gehalt an Stammw ürze zwischen 6,4 und 10,1\%, während im Frieden der Vergärungsgrad zwischen 45 und 50 , der Alkoholgehalt $z$ wischen 3,0 und 3,5 , der Gehalt an Stammwürze zwischen 12 und $14 \%$ schwankte. - Zur Unterdrückung der Unterschiebungen von gewöhnlichem für Münchner Bıer wurden von Dr. Mezger geheime Probenentnahmen mit gleichzeitigen polizeilichen Feststellungen bezüglich der angestochenen Fässer gelegentlich der Kontrolle der Bierdruckapparate empfohlen.

3. Verwendung von Phosphorsäure an Stelle von Wein-, Zitronen-und Milchsäure für limonadenähnliche Getränke und Fruchtzubereitungen. Referent Dr. Benz. Nach der Aussprache über diesen Gegenstand wurde beschlossen, daß der Referent eine diesbezügliche Anfrage gleichzeitig auch über die Verwendung von Glykolsāure an das Reichsgesundheitsamt richten soll. Dr. Mezger-Stuttgart.

Schlup der Redaltion am 16. August 1916. 